\title{
A Generalized TRL Algorithm for S-Parameter De-Embedding
}

\author{
P. Colestock and M. Foley \\ Fermi National Accelerator Laboratory \\ P.O. Box 500, Batavia, Illinois 60510
}

April 1993 


\section{Disclaimer}

This report was prepared as an account of work sponsored by an agency of the United States Government. Neither the United States Government nor any agency thereof, nor any of their employees, makes any warranty, express or implied, or assumes any legal liability or responsibility for the accuracy, completeness, or usefulness of any information, apparatus, product, or process disclosed, or represents that its use would not infringe privately owned rights. Reference herein to any specific commercial product, process, or service by trade name, trademark, manufacturer, or otherwise, does not necessarily constitute or imply its endorsement, recommendation, or favoring by the United States Government or any agency thereof. The views and opinions of authors expressed herein do not necessarily state or reflect those of the United States Government or any agency thereof. 


\title{
A GENERALIZED TRL ALGORITHM FOR S-PARAMETER DE-EMBEDDING
}

\author{
P. Colestock \\ Fermi National Accelerator Laboratory \\ M. Foley \\ Fermi National Accelerator Laboratory \\ FNAL Technical Memo TM-1781
}

\begin{abstract}
At FNAL bench measurements of the longitudinal impedance of various beamline components have been performed using stretched wire methods. The basic approach is to use a network analyzer (NWA) to measure the transmission and reflection characteristics (s-parameters) of the beam line component. It is then possible to recover the effective longitudinal impedance from the s-parameters. Several NWA calibration procedures have been implemented in an effort to improve the accuracy of these measurements. These procedures are mathematical techniques for extracting the s-parameters of a test device from external NWA measurements which include the effect of measurement fixtures. The TRL algorithm has proven to be the most effective of these techniques. This method has the advantage of properly accounting for the nonideal calibration standards used in the NWA measurements.
\end{abstract}

\section{Introduction}

The objective of this work is to recover an equivalent impedance for a given device-under-test (DUT) using a bi-directional reflectometer, otherwise known 
as a network analyzer (NWA). The basic algorithm consists of applying an incident wave to the DUT, which is characterized as a general two-port network, and measuring the vector voltages scattered into the forward and reverse directions. The resulting data can be used to calculate s-parameters. However, the measurements are complicated by the fact that transitions occur between the NWA and the DUT. The diagram below is a schematic representation of the measurement setup. A and B are general, linear networks representing the errors occuring in the s-parameter measurements of the DUT. The influence of error networks A and B must be removed from the data in order to accurately evaluate the s-parameters of the DUT. Using standard circuit analysis, it is possible to recover the effective longitudinal, as well as transverse, impedance of the DUT from the de-embedded s-parameters.

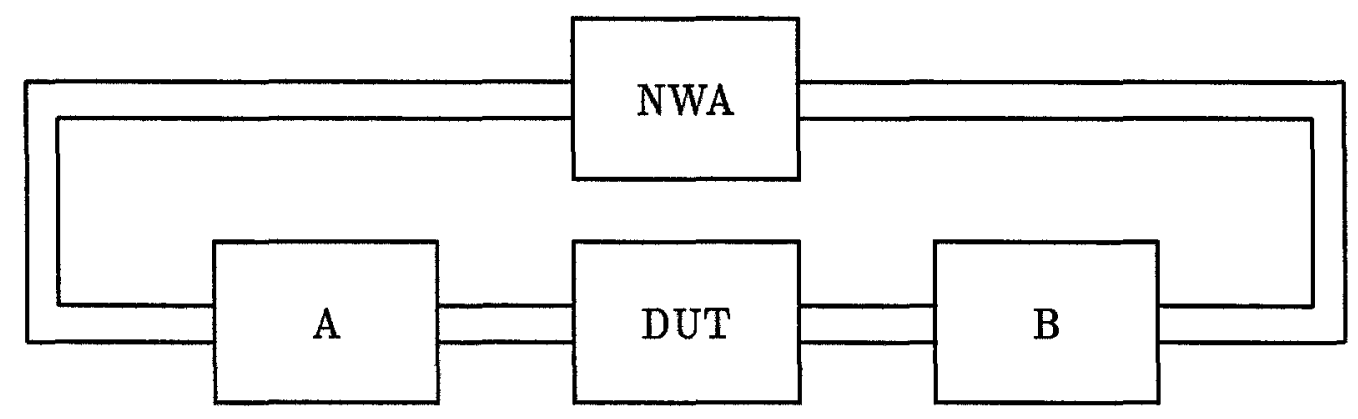

The method described in this Technical Memo is based on a generalization of the Thru-Reflect-Line (TRL) algorithm [1,2]. The calibration standards required are two lengths of transmission line and two shorts with equal reflection coefficient. The lengths of the transmission lines and the value of the reflection coefficient for the shorts are not required to be known. However, the ratio of the lengths of the two transmission lines is required.

Assuming the transmission lines used for calibration are nonreflecting, the s-parameter matrices for line 1 and line 2 are defined by

$$
\left[S_{L 1}\right]=\left[\begin{array}{cc}
0 & L_{1}^{+} \\
L_{1}^{+} & 0
\end{array}\right]
$$

and

$$
\left[S_{L 2}\right]=\left[\begin{array}{cc}
0 & L_{2}^{+} \\
L_{2}^{+} & 0
\end{array}\right]
$$


The s-parameter matrix for both shorts is

$$
\left[S_{S H O R T}\right]=\left[\begin{array}{ll}
\gamma & 0 \\
0 & \gamma
\end{array}\right]
$$

\section{Derivation of Equations Relating S-Parameters Measured at NWA Ports to S-Parameters of Test Device}

The object of this section is to find the expressions which relate the sparameters of the DUT, $S_{i j}$, to the NWA measurements, $S_{i j m}$, where it is assumed that the s-parameters of the networks $A$ and $B$ have been determined, including the complex phase factor $L_{1}^{+}$. In Section 4 the expressions associated with the TRL calibration method which yield these network sparameters are derived.

The network flow graph for the generalized TRL calibration is shown in Figure 1. The reference planes for this calibration method are located at the middle of the shorter transmission line. Therefore, half the length of the shorter line is included on each side of the DUT.

In order to develop expressions relating the s-parameters measured at the NWA ports, $S_{i j m}$, to the s-parameters of the DUT, $S_{i j}$, one follows the procedure of $[3,4]$. From the network flow graph in Figure 1:

$$
\begin{aligned}
& b_{0}=S_{11 A} a_{0}+S_{12 A} a_{1} \\
& b_{1}=S_{21 A} a_{0}+S_{22 A} a_{1} \\
& a_{1}=L_{1}^{+} S_{11} b_{1}+L_{1}^{+} S_{12} b_{2} \\
& a_{2}=L_{1}^{+} S_{21} b_{1}+L_{1}^{+} S_{22} b_{2} \\
& b_{2}=S_{11 B} a_{2}+S_{12 B} a_{3} \\
& b_{3}=S_{21 B} a_{2}+S_{22 B} a_{3}
\end{aligned}
$$

By definition, the s-parameters measured at the NWA ports are:

$$
S_{11 m}=\left.\frac{b_{0}}{a_{0}}\right|_{a_{3}=0}
$$




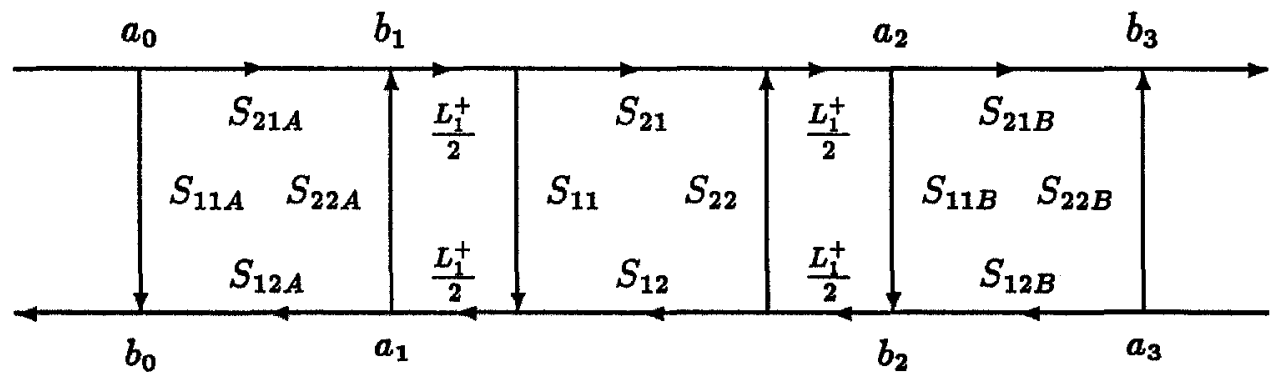

Figure 1: Network Flow Graph for Generalized TRL Calibration

and

$$
S_{21 m}=\left.\frac{b_{3}}{a_{0}}\right|_{a_{3}=0}
$$

From (8) and (9), for $S_{11 m}$ and $S_{21 m}\left(a_{3}=0\right)$.

$$
\begin{aligned}
& b_{2}=S_{11 B} a_{2} \\
& b_{3}=S_{21 B} a_{2}
\end{aligned}
$$

Therefore,

$$
a_{2}=\frac{b_{3}}{S_{21 B}}
$$

From (10) and (12),

$$
b_{2}=\frac{S_{11 B}}{S_{21 B}} b_{3}
$$

From (6)and (13),

$$
a_{1}=L_{1}^{+} S_{11} b_{1}+L_{1}^{+} S_{12} \frac{S_{11 B}}{S_{21 B}} b_{3}
$$

From (5) and (14),

$$
a_{1}=L_{1}^{+} S_{11}\left(S_{21 A} a_{0}+S_{22 A} a_{1}\right)+L_{1}^{+} S_{12} \frac{S_{11 B}}{S_{21 B}} b_{3}
$$


and

$$
a_{1}\left(1-L_{1}^{+} S_{11} S_{22 A}\right)=L_{1}^{+} S_{11} S_{21 A} a_{0}+L_{1}^{+} S_{12} \frac{S_{11 B}}{S_{21 B}} b_{3}
$$

From (4) and (16),

$$
\begin{aligned}
\left(1-L_{1}^{+} S_{11} S_{22 A}\right) b_{0}= & S_{11 A}\left(1-L_{1}^{+} S_{11} S_{22 A}\right) a_{0}+ \\
& L_{1}^{+} S_{11} S_{12 A} S_{21 A} a_{0}+L_{1}^{+} S_{12} \frac{S_{12 A} S_{11 B}}{S_{21 B}} b_{3}
\end{aligned}
$$

Dividing through both sides of (17) by $a_{0}$ yields,

$$
\begin{aligned}
\left(1-L_{1}^{+} S_{11} S_{22 A}\right) S_{11 m}= & S_{11 A}\left(1-L_{1}^{+} S_{11} S_{22 A}\right)+ \\
& L_{1}^{+} S_{11} S_{12 A} S_{21 A}+L_{1}^{+} S_{12} \frac{S_{12 A} S_{11 B}}{S_{21 B}} S_{21 m}
\end{aligned}
$$

and

$$
\begin{aligned}
S_{11 m}= & S_{11 A}+L_{1}^{+} S_{11}\left(S_{12 A} S_{21 A}+S_{22 A} S_{11 m}-S_{11 A} S_{22 A}\right)+ \\
& L_{1}^{+} S_{12} \frac{S_{12 A} S_{11 B}}{S_{21 B}} S_{21 m}
\end{aligned}
$$

Multiplying the last term in (19) by $\frac{L_{1}^{+} S_{21 A}}{L_{1}^{+} S_{21 A}}$ and simplifying, one obtains,

$$
\begin{aligned}
& \left(S_{11 m}-S_{11 A}\right) S_{21 A} S_{21 B} L_{1}^{+}= \\
& \quad\left(S_{12 A} S_{21 A} L_{1}^{+}+S_{22 A} L_{1}^{+} S_{11 m}-S_{11 A} S_{22 A} L_{1}^{+}\right) S_{21 A} S_{21 B} L_{1}^{+} S_{11}+ \\
& \quad S_{12 A} S_{21 A} L_{1}^{+} S_{11 B} L_{1}^{+} S_{21 m} S_{12}
\end{aligned}
$$

In order to determine $S_{22 m}$ in terms of the s-parameters of the DUT, make the following substitutions in (20):

$\begin{array}{cc}\text { Replace } & B y \\ S_{11 A} & S_{22 B} \\ S_{12 A} & S_{21 B} \\ S_{21 A} & S_{12 B} \\ S_{22 A} & S_{11 B} \\ S_{11 B} & S_{22 A} \\ S_{21 B} & S_{12 A} \\ S_{11 m} & S_{22 m} \\ S_{21 m} & S_{12 m} \\ S_{11} & S_{22} \\ S_{12} & S_{21}\end{array}$


Equation (20) becomes,

$$
\begin{aligned}
& \left(S_{22 m}-S_{22 B}\right) S_{12 A} S_{12 B} L_{1}^{+}= \\
& \quad\left(S_{12 B} S_{21 B} L_{1}^{+}+S_{11 B} L_{1}^{+} S_{22 m}-S_{11 B} S_{22 B} L_{1}^{+}\right) S_{12 A} S_{12 B} L_{1}^{+} S_{22}+ \\
& \quad S_{12 B} S_{21 B} L_{1}^{+} S_{22 A} L_{1}^{+} S_{12 m} S_{21}
\end{aligned}
$$

From (4),

$$
S_{22 A} b_{0}=S_{22 A} S_{11 A} a_{0}+S_{22 A} S_{12 A} a_{1}
$$

From (5),

$$
S_{12 A} b_{1}=S_{12 A} S_{21 A} a_{0}+S_{12 A} S_{22 A} a_{1}
$$

From (22) and (23),

$$
S_{22 A} b_{0}-S_{22 A} S_{11 A} a_{0}=S_{12 A} b_{1}-S_{12 A} S_{21 A} a_{0}
$$

From (24),

$$
S_{22 A} b_{0}+\left(S_{12 A} S_{21 A}-S_{11 A} S_{22 A}\right) a_{0}=S_{12 A} b_{1}
$$

From (25),

$$
b_{1}=\frac{S_{22 A}}{S_{12 A}} b_{0}+\left(S_{21 A}-\frac{S_{11 A} S_{22 A}}{S_{12 A}}\right) a_{0}
$$

From (26) and (7),

$$
a_{2}=L_{1}^{+} S_{21} \frac{S_{22 A}}{S_{12 A}} b_{0}+L_{1}^{+} S_{21}\left(S_{21 A}-\frac{S_{11 A} S_{22 A}}{S_{12 A}}\right) a_{0}+L_{1}^{+} S_{22} b_{2}
$$

From (27) and (10),

$$
\begin{aligned}
\left(1-L_{1}^{+} S_{11 B} S_{22}\right) a_{2}= & L_{1}^{+} S_{21} \frac{S_{22 A}}{S_{12 A}} b_{0}+ \\
& L_{1}^{+} S_{21}\left(S_{21 A}-\frac{S_{11 A} S_{22 A}}{S_{12 A}}\right) a_{0}
\end{aligned}
$$

From (28) and (12),

$$
\begin{aligned}
\frac{\left(1-L_{1}^{+} S_{11 B} S_{22}\right)}{S_{21 B}} b_{3}= & L_{1}^{+} S_{21} \frac{S_{22 A}}{S_{12 A}} b_{0}+ \\
& L_{1}^{+} S_{21}\left(S_{21 A}-\frac{S_{11 A} S_{22 A}}{S_{12 A}}\right) a_{0}
\end{aligned}
$$


Dividing through both sides of (29) by $a_{0}$ yields,

$$
\begin{aligned}
\frac{\left(1-L_{1}^{+} S_{11 B} S_{22}\right)}{S_{21 B}} S_{21 m}= & L_{1}^{+} S_{21} \frac{S_{22 A}}{S_{12 A}} S_{11 m}+ \\
& L_{1}^{+} S_{21}\left(S_{21 A}-\frac{S_{11 A} S_{22 A}}{S_{12 A}}\right)
\end{aligned}
$$

and

$$
\begin{aligned}
S_{21 m}= & L_{1}^{+} S_{21} S_{21 A} S_{21 B}\left(1+\frac{S_{22 A}}{S_{12 A} S_{21 A}} S_{11 m}-\frac{S_{11 A} S_{22 A}}{S_{12 A} S_{21 A}}\right)+ \\
& L_{1}^{+} S_{22} S_{11 B} S_{21 m}
\end{aligned}
$$

Multiplying both sides of (31) by $S_{12 A} s_{21 A} L_{1}^{+}$yields,

$$
\begin{aligned}
& S_{21 m} S_{12 A} S_{21 A} L_{1}^{+}= \\
& \quad\left(S_{12 A} S_{21 A} L_{1}^{+}+S_{22 A} L_{1}^{+} S_{11 m}-S_{11 A} S_{22 A} L_{1}^{+}\right) S_{21 A} S_{21 B} L_{1}^{+} S_{21}+ \\
& \quad S_{12 A} S_{21 A} L_{1}^{+} S_{11 B} L_{1}^{+} S_{21 m} S_{22}
\end{aligned}
$$

In order to determine $S_{12 m}$ in terms of the s-parameters of the DUT, make the same substitutions as before in (32):

$$
\begin{aligned}
S_{12 m} & S_{12 B} S_{21 B} L_{1}^{+}= \\
& \left(S_{12 B} S_{21 B} L_{1}^{+}+S_{11 B} L_{1}^{+} S_{22 m}-S_{11 B} S_{22 B} L_{1}^{+}\right) S_{12 A} S_{12 B} L_{1}^{+} S_{12}+ \\
& S_{12 B} S_{21 B} L_{1}^{+} S_{22 A} L_{1}^{+} S_{12 m} S_{11}
\end{aligned}
$$

Equations (20), (21), (32) and (33) relate the s-parameters measured at the NWA ports, $S_{i j m}$, to the s-parameters of the DUT, $S_{i j}$.

\section{Standard NWA Error Model}

In order to find the s-parameters of the error networks A and B, it is useful to define a set of error terms which represent forward and reverse coupling factors at each network.

The network flow graph of error terms for the generalized TRL calibration is shown in Figure 2. The corresponding error terms are given by [2]: 


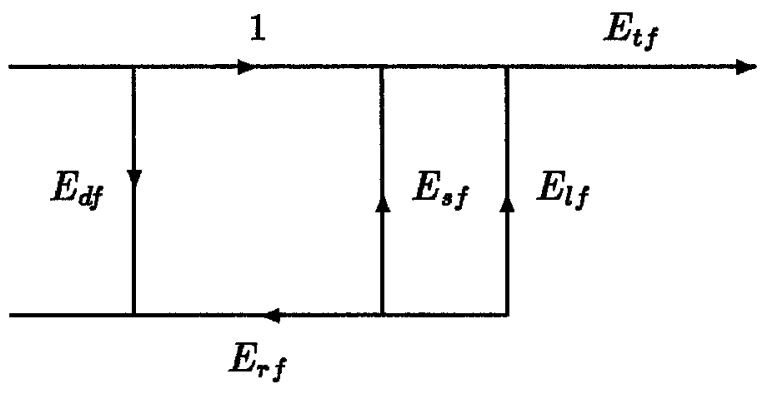

Forward Error Model

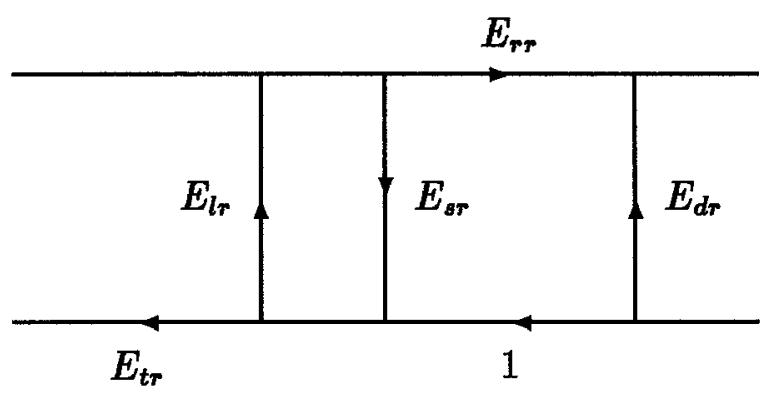

Reverse Error Model

Figure 2: Network Error Model for Generalized TRL Calibration 


$$
\begin{array}{ll}
E_{d f}=S_{11 A} & E_{d r}=S_{22 B} \\
E_{s f}=S_{22 A} L_{1}^{+} & E_{s r}=S_{11 B} L_{1}^{+} \\
E_{r f}=S_{12 A} S_{21 A} L_{1}^{+} & E_{r r}=S_{12 B} S_{21 B} L_{1}^{+} \\
E_{l f}=S_{11 B} L_{1}^{+} & E_{l r}=S_{22 A} L_{1}^{+} \\
E_{t f}=S_{21 A} S_{21 B} L_{1}^{+} & E_{t r}=S_{12 A} S_{12 B} L_{1}^{+}
\end{array}
$$

Using the error terms defined above, equations (20), (21), (32) and (33) become

$$
\begin{aligned}
& \left(S_{11 m}-E_{d f}\right) E_{t f}=\left(E_{r f}+E_{s f} S_{11 m}-E_{d f} E_{s f}\right) E_{t f} S_{11}+ \\
& E_{r f} E_{l f} S_{21 m} S_{12} \\
& S_{21 m} E_{r f}=\left(E_{r f}+E_{s f} S_{11 m}-E_{d f} E_{s f}\right) E_{t f} S_{21}+ \\
& E_{r f} E_{l f} S_{21 m} S_{22} \\
& \left(S_{22 m}-E_{d r}\right) E_{t r}=\left(E_{r r}+E_{s r} S_{22 m}-E_{d r} E_{s r}\right) E_{t r} S_{22}+ \\
& E_{r r} E_{l r} S_{12 m} S_{21} \\
& S_{12 m} E_{r r}=\left(E_{r r}+E_{s r} S_{22 m}-E_{d r} E_{s r}\right) E_{t r} S_{12}+ \\
& E_{r r} E_{l r} S_{12 m} S_{11}
\end{aligned}
$$

\section{Calculation of Error Terms}

The purpose of this section is to evaluate the error terms defined in the preceding section using a set of calibration standards. First a transmission line is connected between the networks $\mathrm{A}$ and $\mathrm{B}$, and a set of s-parameters are measured at the NWA ports. Then a second transmission line with a known incremental length relative to line 1 is connected and the measurements are repeated. Third, a short with an unknown reflection coefficient is connected at each network in turn, and the reflection coefficients at the NWA are measured. The relevant expressions which yield the error terms defined above 
are derived in this section.

For a general two-port network of the form

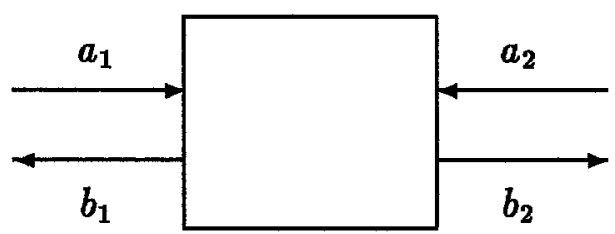

define the wave cascade matrix $[\mathrm{R}]$ by

$$
\left[\begin{array}{l}
b_{1} \\
a_{1}
\end{array}\right]=[R]\left[\begin{array}{l}
a_{2} \\
b_{2}
\end{array}\right]=\left[\begin{array}{ll}
R_{11} & R_{12} \\
R_{21} & R_{22}
\end{array}\right]\left[\begin{array}{l}
a_{2} \\
b_{2}
\end{array}\right]
$$

Note that, in terms of the s-parameters of the two-port network,

$$
[R]=\frac{1}{S_{21}}\left[\begin{array}{cc}
-\Delta & S_{11} \\
-S_{22} & 1
\end{array}\right]
$$

where $\Delta=S_{11} S_{22}-S_{12} S_{21}$.

If the wave cascade matrices of the error boxes $A$ and $B$ are denoted by $\left[R_{A}\right]$ and $\left[R_{B}\right]$ respectively, and those for line 1 and line 2 are $\left[R_{L 1}\right]$ and $\left[R_{L 2}\right]$, then successively connecting line 1 and line 2 between error boxes $A$ and $B$ yields,

$$
\begin{aligned}
& {\left[R_{D 1}\right]=\left[R_{A}\right]\left[R_{L 1}\right]\left[R_{B}\right]} \\
& {\left[R_{D 2}\right]=\left[R_{A}\right]\left[R_{L 2}\right]\left[R_{B}\right]}
\end{aligned}
$$

Note that from (1), (2) and (39),

$$
\left[R_{L 1}\right]=\left[\begin{array}{cc}
L_{1}^{+} & 0 \\
0 & 1 / L_{1}^{+}
\end{array}\right]=\left[\begin{array}{cc}
L_{1}^{+} & 0 \\
0 & L_{1}^{-}
\end{array}\right]
$$

and

$$
\left[R_{L 2}\right]=\left[\begin{array}{cc}
L_{2}^{+} & 0 \\
0 & 1 / L_{2}^{+}
\end{array}\right]=\left[\begin{array}{cc}
L_{2}^{+} & 0 \\
0 & L_{2}^{-}
\end{array}\right]
$$


Eliminating $\left[R_{B}\right]$ from (40) and (41), one obtains

$$
\begin{aligned}
{\left[R_{D 2}\right]\left[R_{D 1}\right]^{-1}\left[R_{A}\right]=} & {\left[R_{A}\right]\left[R_{L 2}\right]\left[R_{B}\right]\left[R_{B}\right]^{-1}\left[R_{L 1}\right]^{-1}\left[R_{A}\right]^{-1}\left[R_{A}\right] } \\
& =\left[R_{A}\right]\left[R_{L 2}\right]\left[R_{L 1}\right]^{-1} \\
& =\left[R_{A}\right][L]
\end{aligned}
$$

where

$$
[L]=\left[R_{L 2}\right]\left[R_{L 1}\right]^{-1}=\left[\begin{array}{cc}
L_{2}^{+} L_{1}^{-} & 0 \\
0 & L_{1}^{+} L_{2}^{-}
\end{array}\right]=\left[\begin{array}{cc}
L^{+} & 0 \\
0 & L^{-}
\end{array}\right]
$$

Defining

$$
[P]=\left[R_{D 2}\right]\left[R_{D 1}\right]^{-1}
$$

equation (44) becomes

$$
[P]\left[R_{A}\right]=\left[R_{A}\right][L]
$$

or

$$
\left[\begin{array}{cc}
P_{11} R_{A 11}+P_{12} R_{A 21} & P_{11} R_{A 12}+P_{12} R_{A 22} \\
P_{21} R_{A 11}+P_{22} R_{A 21} & P_{21} R_{A 12}+P_{22} R_{A 22}
\end{array}\right]=\left[\begin{array}{cc}
L^{+} R_{A 11} & L^{-} R_{A 12} \\
L^{+} R_{A 21} & L^{-} R_{A 22}
\end{array}\right]
$$

Solve for the ratios below using (39) and (46):

$$
\begin{aligned}
& \frac{R_{A 11}}{R_{A 21}}=\frac{-P_{12}}{P_{11}-L^{+}}=\frac{L^{+}-P_{22}}{P_{21}}=\frac{\Delta}{S_{22 A}}=S_{11 A}-\frac{S_{12 A} S_{21 A}}{S_{22 A}} \\
& \frac{R_{A 12}}{R_{A 22}}=\frac{-P_{12}}{P_{11}-L^{-}}=\frac{L^{-}-P_{22}}{P_{21}}=S_{11 A}
\end{aligned}
$$

Eliminating $\left[R_{A}\right]$ from (40) and (41) following a procedure similar to that above, one obtains,

$$
\left[R_{B}\right][Q]=[L]\left[R_{B}\right]
$$

where $[Q]=\left[R_{D 1}\right]^{-1}\left[R_{D 2}\right]$, and

$$
\begin{aligned}
& \frac{R_{B 11}}{R_{B 12}}=\frac{-Q_{21}}{Q_{11}-L^{+}}=\frac{L^{+}-Q_{22}}{Q_{12}}=-\frac{\Delta}{S_{11 B}}=-S_{22 B}+\frac{S_{12 B} S_{21 B}}{S_{11 B}} \\
& \frac{R_{B 21}}{R_{B 22}}=\frac{-Q_{21}}{Q_{11}-L^{-}}=\frac{L^{-}-Q_{22}}{Q_{12}}=-S_{22 B}
\end{aligned}
$$


From (47)-(50),

$$
\begin{aligned}
\left(L^{+}\right)^{2}-\left(P_{11}+P_{22}\right) L^{+}+\Delta P & =0 \\
\left(L^{-}\right)^{2}-\left(P_{11}+P_{22}\right) L^{-}+\Delta P & =0 \\
\left(L^{+}\right)^{2}-\left(Q_{11}+Q_{22}\right) L^{+}+\Delta Q & =0 \\
\left(L^{-}\right)^{2}-\left(Q_{11}+Q_{22}\right) L^{-}+\Delta Q & =0
\end{aligned}
$$

where $\Delta P=P_{11} P_{22}-P_{12} P_{21}$ and $\Delta Q=Q_{11} Q_{22}-Q_{12} Q_{21}$. Note that

$$
\Delta P=\left[R_{D 2}\right]\left[R_{D 1}\right]^{-1}=\left[R_{D 1}\right]^{-1}\left[R_{D 2}\right]=\Delta Q
$$

Subtracting (53) from (51) or (54) from (52) implies,

$$
P_{11}+P_{22}=Q_{11}+Q_{22}
$$

Therefore, $L^{+}$and $L^{-}$are the two roots of the quadratic equation

$$
\left(L^{ \pm}\right)^{2}-\operatorname{Tr} P L^{ \pm}+\Delta P=0
$$

In the idealized case where there are no losses, $L^{+}$and $L^{-}$form a conjugate pair of roots.

Equation (55) can be solved and the ratios (47)-(50) evaluated if the elements of $[P]$ and $[Q]$ are known. These are determined from the NWA s-parameter measurements made by successively connecting line 1 and line 2 between error boxes A and B. This procedure is illustrated in Appendix A.

Now insert a short with unknown reflection coefficient, $\gamma$, at each reference plane, as shown in Figure 3 and Figure 4.

From the diagram in Figure 3,

$$
\left[\begin{array}{c}
\rho_{A} \\
1
\end{array}\right]=\left[\begin{array}{ll}
R_{A 11} & R_{A 12} \\
R_{A 21} & R_{A 22}
\end{array}\right]\left[\begin{array}{c}
\gamma b_{2} \\
b_{2}
\end{array}\right]
$$

Therefore,

$$
\begin{gathered}
\rho_{A}=\left(\gamma R_{A 11}+R_{A 12}\right) b_{2} \\
1=\left(\gamma R_{A 21}+R_{A 22}\right) b_{2}
\end{gathered}
$$




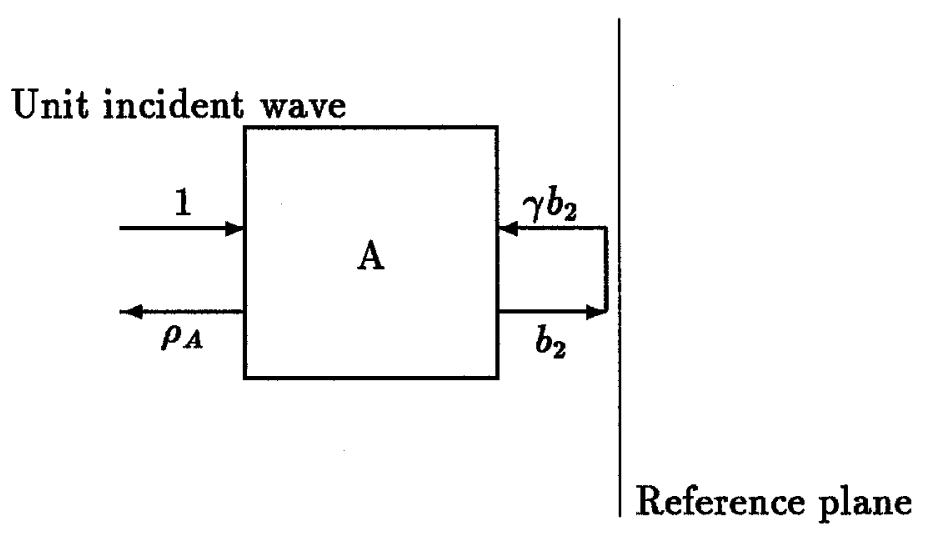

Figure 3: Reflect at reference plane of DUT

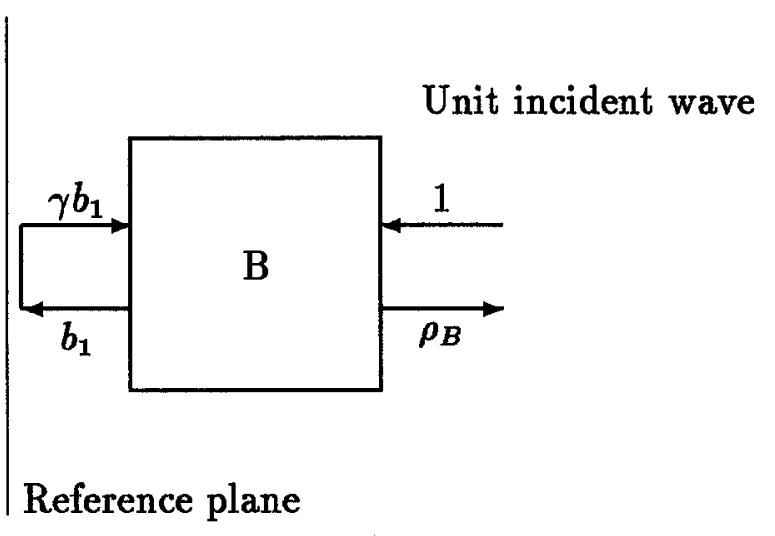

Figure 4: Reflect at reference plane of DUT 
Eliminating $b_{2}$ from (56) and (57), one obtains

$$
\rho_{A}\left(\gamma R_{A 21}+R_{A 22}\right)=\gamma R_{A 11}+R_{A 12}
$$

From the diagram in Figure 4,

$$
\left[\begin{array}{c}
b_{1} \\
\gamma b_{1}
\end{array}\right]=\left[\begin{array}{ll}
R_{B 11} & R_{B 12} \\
R_{B 21} & R_{B 22}
\end{array}\right]\left[\begin{array}{c}
1 \\
\rho_{B}
\end{array}\right]
$$

Therefore,

$$
\begin{aligned}
& b_{1}=R_{B 11}+\rho_{B} R_{B 22} \\
& \gamma b_{1}=R_{B 21}+\rho_{B} R_{B 22}
\end{aligned}
$$

Eliminating $b_{1}$ from (59) and (60), one obtains

$$
\gamma\left(R_{B 11}+\rho_{B} R_{B 22}\right)=R_{B 21}+\rho_{B} R_{B 22}
$$

Eliminating $\gamma$ from (58) and (61), one obtains

$$
\alpha \frac{R_{A 22}}{R_{A 21}}=\beta \frac{R_{B 22}}{R_{B 12}}
$$

where

$$
\alpha=\frac{\frac{R_{A 12}}{R_{A 22}}-\rho_{A}}{\rho_{A}-\frac{R_{A 11}}{R_{A 21}}}
$$

and

$$
\beta=\frac{\frac{R_{B 21}}{R_{B 22}}+\rho_{B}}{\rho_{B}+\frac{R_{B 11}}{R_{B 12}}}
$$

Note that $\rho_{A}$ and $\rho_{B}$ are known from the NWA measurements made by successively inserting short 1 and short 2 at each reference plane.

Consider the NWA measurement with line 1 inserted. The reflection coefficient for this measurement is:

$$
\begin{aligned}
S_{11 D 1} & \doteq \frac{R_{D 1}^{12}}{R_{D 1}^{22}}=\frac{R_{A 11} R_{B 12} L_{1}^{+}+R_{A 12} R_{B 22} L_{1}^{-}}{R_{A 21} R_{B 12} L_{1}^{+}+R_{A 22} R_{B 22} L_{1}^{-}} \\
& =\frac{\frac{R_{A 11}}{R_{A 21}}+\frac{R_{A 12}}{R_{A 21}} \frac{R_{B 22}}{R_{B 12}} \frac{L_{1}^{-}}{L_{1}^{+}}}{1+\frac{R_{A 22}}{R_{A 21}} R_{B 22} R_{12}^{-}}
\end{aligned}
$$


From (62),

$$
\frac{R_{B 22}}{R_{B 12}}=\frac{\alpha}{\beta} \frac{R_{A 22}}{R_{A 21}}
$$

Substituting in (65), one obtains

$$
\begin{aligned}
& S_{11 D 1}= \frac{\frac{R_{A 11}}{R_{A 21}}+\left(\frac{\alpha}{\beta}\right)\left(\frac{L_{1}^{-}}{L_{1}^{+}}\right) \frac{R_{A 12}}{R_{A 21}} \frac{R_{A 22}}{R_{A 21}}}{1+\left(\frac{\alpha}{\beta}\right)\left(\frac{L_{1}^{-}}{L_{1}^{+}}\right) \frac{R_{A 22}}{R_{A 21}}} \\
&= \frac{\frac{R_{A 22}}{R_{A 21}}}{R_{A 21}}+\left(\frac{\alpha}{\beta}\right)\left(\frac{L_{1}^{-}}{L_{1}^{+}}\right) \frac{R_{A 12}}{R_{A 22}}\left(\frac{R_{A 11}}{R_{A 21}}\right)^{2}\left(\frac{R_{A 22}}{R_{A 11}}\right)^{2} \\
& 1+\left(\frac{\alpha}{\beta}\right)\left(\frac{L_{1}^{-}}{L_{1}^{+}}\right)\left(\frac{R_{A 11}}{R_{A 21}}\right)^{2}\left(\frac{R_{A 22}}{R_{A 11}}\right)^{2}
\end{aligned}
$$

From (66) the ratio $\frac{R_{A 22}}{R_{A 11}}$ can be determined

$$
\frac{R_{A 22}}{R_{A 11}}= \pm \sqrt{\frac{\frac{1}{S_{11 D 1}} \frac{R_{A 11}}{R_{21}}-1}{\left(1-\frac{1}{S_{11 D 1}} \frac{R_{A 12}}{R_{A 22}}\right)\left(\frac{\alpha}{\beta}\right)\left(\frac{L_{1}^{-}}{L_{1}^{+}}\right)\left(\frac{R_{A 11}}{R_{A 21}}\right)^{2}}}
$$

Using (62) the ratio $\frac{R_{B 22}}{R_{B 11}}$ can be determined

$$
\frac{R_{B 22}}{R_{B 11}}=\frac{\frac{R_{B 22}}{R_{B 12}}}{\frac{R_{B 11}}{R_{B 12}}}=\frac{\frac{\alpha}{\beta} \frac{R_{A 22}}{R_{A 21}}}{\frac{R_{B 11}}{R_{B 12}}}=\frac{\alpha}{\beta} \frac{\frac{R_{A 11}}{R_{A 21}}}{\frac{R_{B 11}}{R_{B 12}}} \frac{R_{A 22}}{R_{A 11}}
$$

In order to evaluate (67), (68) and the error terms in (34)-(37), the values of $L_{1}^{+}$and $L_{1}^{-}$must be calculated. Defining

$$
\xi \doteq \frac{L_{2}}{L_{1}} \quad \text { where } \quad L_{2}>L_{1}
$$

as the ratio of the two lengths of transmission line, it is shown in Appendix B that

$$
L_{1}^{-}=\left(L^{-}\right)^{\frac{1}{(\xi-1)}}
$$

and

$$
L_{1}^{+}=\left(L^{+}\right)^{\frac{1}{(\xi-1)}}
$$

where $L^{+}$and $L^{-}$are the two roots of (55). 
It is also necessary to select the proper root when evaluating (67) and (68). This is accomplished by estimating the phase of the reflect, as shown in Appendix C. Physically, the selection corresponds to distinguishing between an open or a short at the reference plane of the DUT.

Using (39),

$$
\begin{aligned}
& S_{11 A}=S_{21 A} R_{A 12}=\frac{R_{A 12}}{R_{A 22}} \\
& S_{22 A}=-S_{21 A} R_{A 21}=-\frac{R_{A 21}}{R_{A 22}}=-\frac{R_{A 21}}{R_{A 11}} \frac{R_{A 11}}{R_{A 22}}
\end{aligned}
$$

and

$$
S_{12 A} S_{21 A}=S_{21 A} R_{A 11}+S_{11 A} S_{22 A}=S_{11 A} S_{22 A}+\frac{R_{A 11}}{R_{A 22}}
$$

From (69), (70) and (71), one obtains

$$
\begin{aligned}
S_{12 A} S_{21 A} & =\frac{R_{A 12}}{R_{A 22}}\left(-\frac{R_{A 21}}{R_{A 11}} \frac{R_{A 11}}{R_{A 22}}\right)+\frac{R_{A 11}}{R_{A 22}} \\
& =\frac{R_{A 11}}{R_{A 22}}-\frac{R_{A 11}}{R_{A 22}} \frac{R_{A 12}}{R_{A 22}} \frac{R_{A 21}}{R_{A 11}}
\end{aligned}
$$

Similarly,

$$
\begin{aligned}
S_{11 B} & =\frac{R_{B 12}}{R_{B 11}} \frac{R_{B 11}}{R_{B 22}} \\
S_{22 B} & =-\frac{R_{B 21}}{R_{B 22}}
\end{aligned}
$$

and

$$
S_{21 B} S_{12 B}=\frac{R_{B 11}}{R_{B 22}}-\frac{R_{B 11}}{R_{B 22}} \frac{R_{B 21}}{R_{B 22}} \frac{R_{B 12}}{R_{B 11}}
$$

The transmission coefficient for the NWA measurement with line 1 inserted is given by

$$
\begin{aligned}
S_{21 D 1} \doteq \frac{1}{R_{D 1}^{22}} & =\frac{1}{R_{A 21} R_{B 12} L_{1}^{+}+R_{A 22} R_{B 22} L_{1}^{-}} \\
& \left.=\frac{1}{R_{A 21} R_{B 12} L_{1}^{+}\left[1+\frac{R_{A 22}}{R_{A 21}} R_{B 22}\right.}\left(\frac{L_{1}^{-}}{L_{1}^{+}}\right)\right]
\end{aligned}
$$


From (39) and (62),

$$
\begin{aligned}
S_{21 D 1} & =\frac{1}{-\frac{S_{22 A}}{S_{21 A}} \frac{S_{11 B}}{S_{21 B}} L_{1}^{+}\left[1+\left(\frac{\alpha}{\beta}\right)\left(\frac{L_{1}^{-}}{L_{1}^{+}}\right)\left(\frac{R_{A 22}}{R_{A 21}}\right)^{2}\right]} \\
& =\frac{S_{21 A} S_{21 B}}{-S_{22 A} S_{11 B} L_{1}^{+}}\left[1+\left(\frac{\alpha}{\beta}\right)\left(\frac{L_{1}^{-}}{L_{1}^{+}}\right)\left(\frac{R_{A 11}}{R_{A 21}}\right)^{2}\left(\frac{R_{A 22}}{R_{A 11}}\right)^{2}\right]
\end{aligned}
$$

From(77),

$$
S_{21 A} S_{21 B}=-S_{21 D 1} S_{22 A} S_{11 B} L_{1}^{+}\left[1+\left(\frac{\alpha}{\beta}\right)\left(\frac{L_{1}^{-}}{L_{1}^{+}}\right)\left(\frac{R_{A 11}}{R_{A 21}}\right)^{2}\left(\frac{R_{A 22}}{R_{A 11}}\right)^{2}\right]
$$

From (40),

$$
\left[R_{D 1}\right]=\left[R_{A}\right]\left[R_{L 1}\right]\left[R_{B}\right]
$$

Therefore,

$$
\left|R_{D 1}\right|=\left|R_{A}\right|\left|R_{L 1}\right|\left|R_{B}\right|=\frac{S_{12 A}}{S_{21 A}} \frac{S_{12 B}}{S_{21 B}} L_{1}^{+} L_{1}^{-}
$$

By definition,

$$
L_{1}^{+} L_{1}^{-}=L_{1}^{+} \frac{1}{L_{1}^{+}}=1
$$

From (79) and (80),

$$
S_{12 A} S_{12 B}=\left|R_{D 1}\right| S_{21 A} S_{21 B}=\frac{S_{12 D 1}}{S_{21 D 1}} S_{21 A} S_{21 B}
$$

From (78) and (81),

$$
S_{12 A} S_{12 B}=-S_{12 D 1} S_{22 A} S_{11 B} L_{1}^{+}\left[1+\left(\frac{\alpha}{\beta}\right)\left(\frac{L_{1}^{-}}{L_{1}^{+}}\right)\left(\frac{R_{A 11}}{R_{A 21}}\right)^{2}\left(\frac{R_{A 22}}{R_{A 11}}\right)^{2}\right]
$$

From (69)-(75), (70) and (82) the relevant s-parameters of error networks A and $B$ are expressed in terms of known ratios, and therefore the error terms in equations (34)-(37) can be evaluated. 


\section{De-Embedding the S-Parameters of the Test Device}

The purpose of this section is to summarize the results obtained thus far, and give the expressions which show how to de-embed the s-parameters of the DUT from the NWA measurements.

Equations (34)-(37) are expressed in the form

$$
\begin{gathered}
A_{1}=A_{2} S_{11}+A_{3} S_{12} \\
B_{1}=B_{2} S_{21}+B_{3} S_{22} \\
C_{1}=C_{2} S_{22}+C_{3} S_{21} \\
D_{1}=D_{2} S_{12}+D_{3} S_{11}
\end{gathered}
$$

where

$$
\begin{aligned}
& A_{1}=\left(S_{11 m}-E_{d f}\right) E_{t f} \\
& A_{2}=\left(E_{r f}+E_{s f} S_{11 m}-E_{d f} E_{s f}\right) E_{t f} \\
& A_{3}=S_{21 m} E_{r f} E_{l f} \\
& B_{1}=S_{21 m} E_{r f} \\
& B_{2}=\left(E_{r f}+E_{s f} S_{11 m}-E_{d f} E_{s f}\right) E_{t f} \\
& B_{3}=S_{21 m} E_{r f} E_{l f} \\
& C_{1}=\left(S_{22 m}-E_{d r}\right) E_{t r} \\
& C_{2}=\left(E_{r r}+E_{s r} S_{22 m}-E_{d r} E_{s r}\right) E_{t r} \\
& C_{3}=S_{12 m} E_{r r} E_{l r} \\
& D_{1}=S_{12 m} E_{r r} \\
& D_{2}=\left(E_{r r}+E_{s r} S_{22 m}-E_{d r} E_{s r}\right) E_{t r} \\
& D_{3}=S_{12 m} E_{r r} E_{l r}
\end{aligned}
$$

The s-parameters of the test device are obtained by solving the linear, simultaneous equations (83)-(86) to yield,

$$
S_{11}=\frac{A_{1} D_{2}-D_{1} A_{3}}{A_{2} D_{2}-D_{3} A_{3}}
$$




$$
\begin{gathered}
S_{12}=\frac{A_{2} D_{1}-D_{3} A_{1}}{A_{2} D_{2}-D_{3} A_{3}} \\
S_{21}=\frac{B_{1} C_{2}-C_{1} B_{3}}{B_{2} C_{2}-C_{3} B_{3}} \\
S_{22}=\frac{B_{2} C_{1}-C_{3} B_{1}}{B_{2} C_{2}-C_{3} B_{3}}
\end{gathered}
$$

A listing of the FORTRAN code to implement the generalized TRL algorithm is given in Appendix D.

\section{Calculation of Impedance from De-Embedded S-Parameters}

It is worthwhile to note the relationship between the s-parameters just found, and the concept of shunt impedance which is usually applied to beamline components.

The relation between the scattering matrix, $[\mathrm{S}]$, and the impedance matrix, $[\mathrm{Z}]$, for the DUT is given by $[5]$,

$$
[S]=([Z]+[I])^{-1}([Z]-[I])
$$

From (87),

$$
\begin{gathered}
([Z]+[I])[S]=[Z]-[I] \\
{[Z]-[Z][S]=[S]+[I]} \\
{[Z]([I]-[S])=[S]+[I]}
\end{gathered}
$$

and therefore

$$
[Z]=([S]+[I])([I]-[S])^{-1}
$$


Consider the equivalent networks

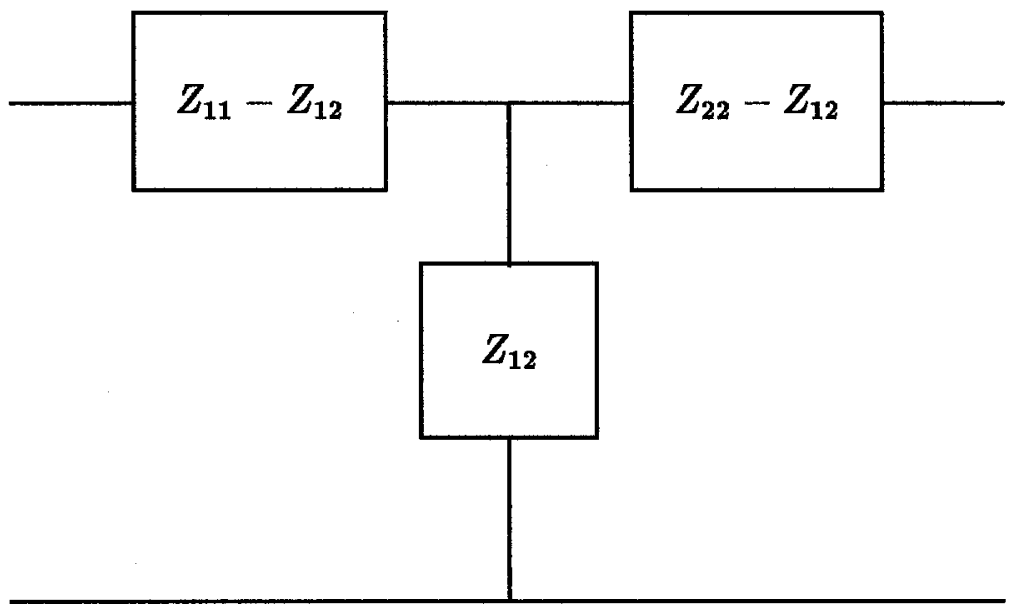

and

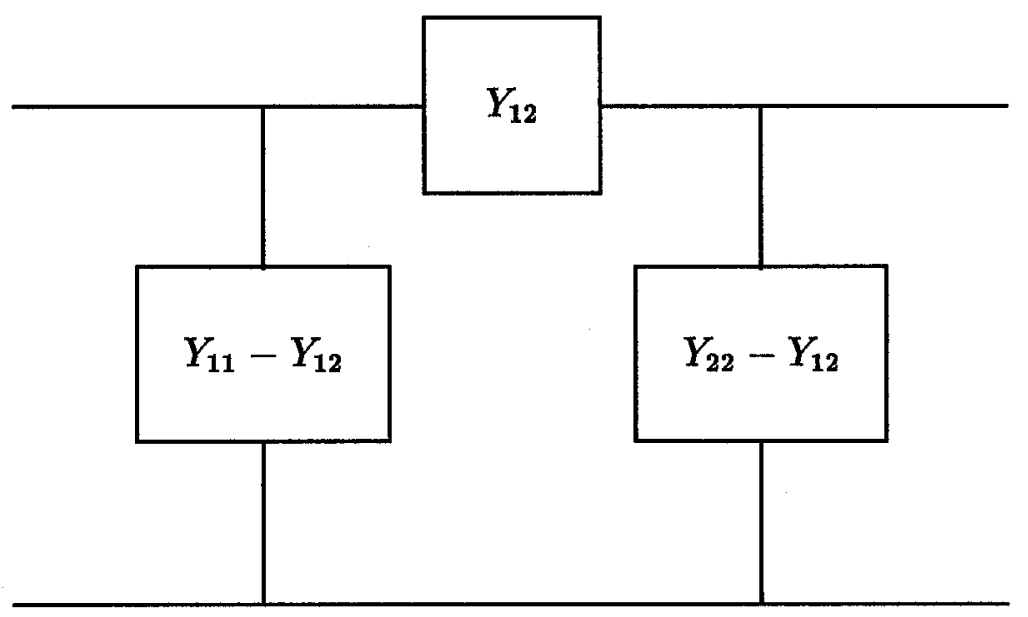

Assuming shunt losses are not negligible, interpret the longitudinal impedance 
as

$$
\frac{Z}{Z_{0}}=\frac{1}{Y_{12}}
$$

By definition, the admittance matrix, [Y], is given by

$$
[Y]=[Z]^{-1}
$$

From (88) and (89),

$$
\begin{aligned}
{[Y]=} & ([I]-[S])([S]+[I])^{-1} \\
= & \frac{1}{1+S_{11}+S_{22}+\Delta}\left[\begin{array}{cc}
1-S_{11} & -S_{12} \\
-S_{21} & 1-S_{22}
\end{array}\right] . \\
& {\left[\begin{array}{cc}
S_{22}+1 & -S_{12} \\
-S_{21} & S_{11}+1
\end{array}\right] }
\end{aligned}
$$

where $\Delta=S_{11} S_{22}-S_{12} S_{21}$.

From (90),

$$
Y_{12}=\frac{2 S_{12}}{1+S_{11}+S_{22}+\Delta}=\frac{2 S_{21}}{1+S_{11}+S_{22}+\Delta}=Y_{21}
$$

Therefore, the impedance, $\mathrm{Z}$, is given by

$$
Z=\frac{Z_{0}\left(1+S_{11}+S_{22}+\Delta\right)}{2 S_{21}}
$$

A listing of the FORTRAN code to implement (91) is given in Appendix E. 


\section{Conclusions}

An algorithm has been derived for de-embedding the impedance parameters of a general 2-port network from a realistic set of s-parameter measurements including the effects of external impedance transformations. The method requires the separate measurement of inserted delays of two different lengths (optimally different by $\lambda / 2$ ), and the measurement of identical, but possibly nonideal, reflects. Moreover, the algorithm has been implemented in the form of a FORTRAN computer code, which can be used with standard NWA output data to provide comparatively accurate values for the de-embedded impedance of a given device over as much as an octave in frequency. This method has the advantage of properly taking into account the often-experienced nonideal transmission line standards encountered in these measurements. The details of the comparison of this algorithm with synthesized data, as well as with an actual device whose impedance is known theoretically, are covered in a separate document [6]. 


\section{References}

[1] G.F. Engen and C.A. Hoer, "Thru-Reflect-Line: An Improved Technique for Calibrating the Dual Six-Port Automatic Network Analyzer", IEEE Transactions on Microwave Theory and Techniques, Vol. MTT-27, No. 12, pp. 987-993, Dec. 1979.

[2] R.R Pantoja, M.J. Howes, J.R. Richardson and R.D. Pollard, "Improved Calibration and Measurement of the Scattering Parameters of Microwave Integrated Circuits", IEEE Transactions on Microwave Theory and Techniques, Vol. MTT-37, No. 11, pp. 1675-1680, Nov. 1989.

[3] J. Staudinger and W. Seely, "MMIC Tests Improved with Standards on Chip", Microwaves and RF, pp. 107-114, Feb. 1987.

[4] D. McGinnis, Thru-Short-Delay De-Embedding, internal communication, Fermi National Accelerator Laboratory, April 1991.

[5] R.E. Collin, Field Theory of Guided Waves, 2nd Edition, IEEE Press, pp. 191-192, 1991.

[6] E. Barsotti, Jr., P. Colestock, and M. Foley, "A Comparison of ThruShort-Delay and Thru-Reflect-Line De-Embedding", FNAL Technical Memo, TM-1782, April 1993. 

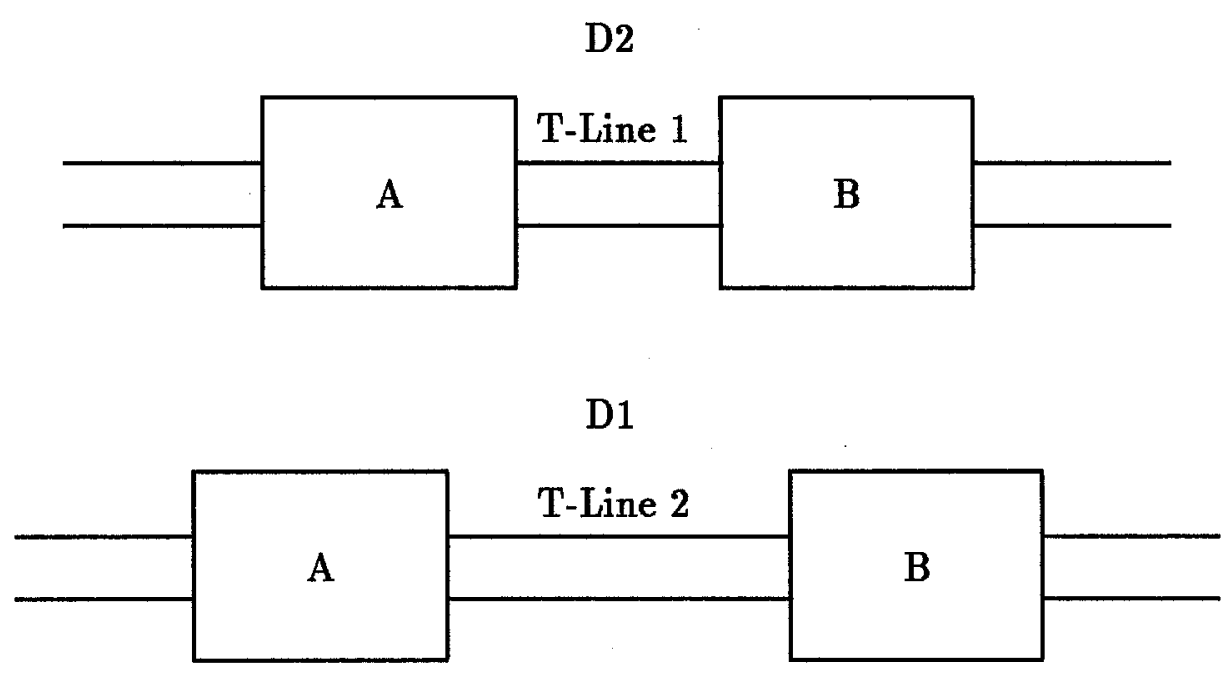

Figure 5: Calibration Networks D1 and D2

\section{Appendix}

\section{A Evaluation of $\mathbf{P}$ and $\mathbf{Q}$ Matrices}

Consider the networks D1 and D2 which are formed by successively connecting transmission line 1 and transmission line 2 between error networks $A$ and $B$, as shown in Figure 5.

NWA measurements on D1 and D2 yield the s-parameter matrices $\left[S_{D 1}\right]$ and 
$\left[S_{D_{2}}\right]$ respectively. Using (39), the corresponding wave cascade matrices are generated,

$$
\begin{aligned}
& {\left[R_{D 1}\right]=\frac{1}{S_{21 D 1}}\left[\begin{array}{cc}
-\Delta_{D 1} & S_{11 D 1} \\
-S_{22 D 1} & 1
\end{array}\right]} \\
& {\left[R_{D 2}\right]=\frac{1}{S_{21 D 2}}\left[\begin{array}{cc}
-\Delta_{D 2} & S_{11 D 2} \\
-S_{22 D 2} & 1
\end{array}\right]}
\end{aligned}
$$

where $\Delta_{D 1}=S_{11 D 1} S_{22 D 1}-S_{12 D 1} S_{21 D 1}$ and $\Delta_{D 2}=S_{11 D 2} S_{22 D 2}-S_{12 D 2} S_{21 D 2}$.

$$
\left[R_{D 1}\right]^{-1}=\frac{1}{S_{12 D 1}}\left[\begin{array}{cc}
1 & -S_{11 D 1} \\
S_{22 D 1} & -\Delta_{D 1}
\end{array}\right]
$$

The elements of $[\mathrm{P}]$ are obtained from

$$
\begin{aligned}
{[P] } & =\left[R_{D 2}\right]\left[R_{D 1}\right]^{-1}=\frac{1}{S_{21 D 2}}\left[\begin{array}{cc}
-\Delta_{D 2} & S_{11 D 2} \\
-S_{22 D 2} & 1
\end{array}\right] \frac{1}{S_{12 D 1}}\left[\begin{array}{cc}
1 & -S_{11 D 1} \\
S_{22 D 1} & -\Delta_{D 1}
\end{array}\right] \\
& =\frac{1}{S_{12 D 1} S_{21 D 2}}\left[\begin{array}{cc}
\left(S_{11 D 2} S_{22 D 1}-\Delta_{D 2}\right) & \left(S_{11 D 1} \Delta_{D 2}-S_{11 D 2} \Delta_{D 1}\right) \\
\left(S_{22 D 1}-S_{22 D 2}\right) & \left(S_{11 D 1} S_{22 D 2}-\Delta_{D 1}\right)
\end{array}\right]
\end{aligned}
$$

Similarly,

$$
\begin{aligned}
{[Q] } & =\left[R_{D 1}\right]^{-1}\left[R_{D 2}\right]=\frac{1}{S_{12 D 1}}\left[\begin{array}{cc}
1 & -S_{11 D 1} \\
S_{22 D 1} & -\Delta_{D 1}
\end{array}\right] \frac{1}{S_{12 D 2}}\left[\begin{array}{cc}
-\Delta_{D 2} & S_{11 D 2} \\
-S_{22 D 2} & 1
\end{array}\right] \\
& =\frac{1}{S_{12 D 1} S_{21 D 2}}\left[\begin{array}{cc}
\left(S_{11 D 1} S_{22 D 2}-\Delta_{D 2}\right) & \left(S_{11 D 2}-S_{11 D 1}\right) \\
\left(S_{22 D 2} \Delta_{D 1}-S_{22 D 1} \Delta_{D 2}\right) & \left(S_{11 D 2} S_{22 D 1}-\Delta_{D 1}\right)
\end{array}\right]
\end{aligned}
$$




\section{B Calculation of $L_{1}^{+}$and $L_{1}^{-}$}

Define $\xi$ as the ratio of the lengths of the two transmission line calibration standards.

$$
\xi \doteq \frac{L_{2}}{L_{1}} \quad \text { where } \quad L_{2}>L_{1}
$$

By definition $L^{+}=L_{2}^{+} L_{1}^{-}$and $L^{-}=L_{1}^{+} L_{2}^{-}$. Therefore,

$$
\begin{aligned}
L^{+} & =e^{-a\left(L_{2}-L_{1}\right)} e^{-j k\left(L_{2}-L_{1}\right)} \\
& =e^{-(a+j k)\left(L_{2}-L_{1}\right)} \\
& =e^{-\sigma\left(L_{2}-L_{1}\right)}
\end{aligned}
$$

and

$$
L^{-}=\frac{1}{L^{+}}=e^{\sigma\left(L_{2}-L_{1}\right)}
$$

where $\sigma=(a+j k), a>0$ is the complex propagation constant for the two transmission line standards. From (92) and (93),

$$
\begin{aligned}
L_{1}^{+} & =e^{-\sigma L_{1}} \\
& =e^{-\sigma\left(L_{2}-L_{1}\right) / \frac{\left(L_{2}-L_{1}\right)}{L_{1}}} \\
& =\left(L^{+}\right)^{\frac{1}{(\xi-1)}}
\end{aligned}
$$

Similarly,

$$
\begin{aligned}
L_{1}^{-} & =e^{\sigma L_{1}} \\
& =e^{\sigma\left(L_{2}-L_{1}\right) / \frac{\left(L_{2}-L_{1}\right)}{L_{1}}} \\
& =\left(L^{-}\right)^{\frac{1}{(\xi-1)}}
\end{aligned}
$$

Therefore,

$$
\begin{aligned}
& L_{1}^{+}=\left(L^{+}\right)^{\frac{1}{(\xi-1)}} \\
& L_{1}^{-}=\left(L^{-}\right)^{\frac{1}{(\xi-1)}}
\end{aligned}
$$




\section{Proper Root Choice}

The proper choice of root in evaluating $\frac{R_{A 22}}{R_{A 11}}$ is accomplished by estimating the phase of the reflect, as shown below.

From (58),

$$
\rho_{A}=\frac{\gamma R_{A 11}+R_{A 12}}{\gamma R_{A 21}+R_{A 22}}=\frac{\gamma \frac{R_{A 11}}{R_{A 22}}+\frac{R_{A 12}}{R_{A 22}}}{\gamma \frac{R_{A 21}}{R_{A 22}}+1}
$$

From (94),

$$
\gamma \frac{R_{A 11}}{R_{A 22}}+\frac{R_{A 12}}{R_{A 22}}=\gamma \rho_{A} \frac{R_{A 21}}{R_{A 22}}+\rho_{A}
$$

and

$$
\gamma\left(\frac{R_{A 11}}{R_{A 22}}-\rho_{A} \frac{R_{A 21}}{R_{A 22}}\right)=\rho_{A}-\frac{R_{A 12}}{R_{A 22}}
$$

Therefore, the unknown reflection coefficient, $\gamma$, is given by

$$
\gamma=\frac{\rho_{A}-\frac{R_{A 12}}{\boldsymbol{R}_{A 22}}}{\frac{\boldsymbol{R}_{A 11}}{\boldsymbol{R}_{A 22}}\left[1-\rho_{a}\left(\frac{\frac{\boldsymbol{R}_{A 21}}{\boldsymbol{R}_{A 21}}}{\boldsymbol{R}_{A 11}}\right)\right]}=\frac{\frac{\boldsymbol{R}_{A 22}}{\boldsymbol{R}_{A 11}}\left[\rho_{A}-\frac{\boldsymbol{R}_{A 12}}{\boldsymbol{R}_{A 22}}\right]}{\left[1-\rho_{A}\left(1 / \frac{\boldsymbol{R}_{A 11}}{\boldsymbol{R}_{A 21}}\right)\right]}
$$

Using (47), (48) and the value of $\rho_{A}$ from NWA measurements, $\gamma$ can be evaluated from (95) for each choice of root in (67). Since $\gamma$ represents the reflection coefficient for a short, the proper root choice is that value of $\frac{R_{A 22}}{R_{A 11}}$ for which the corresponding value of $\gamma$ lies in the shaded region of the complex plane. 


$$
\gamma \text { - Plane }
$$

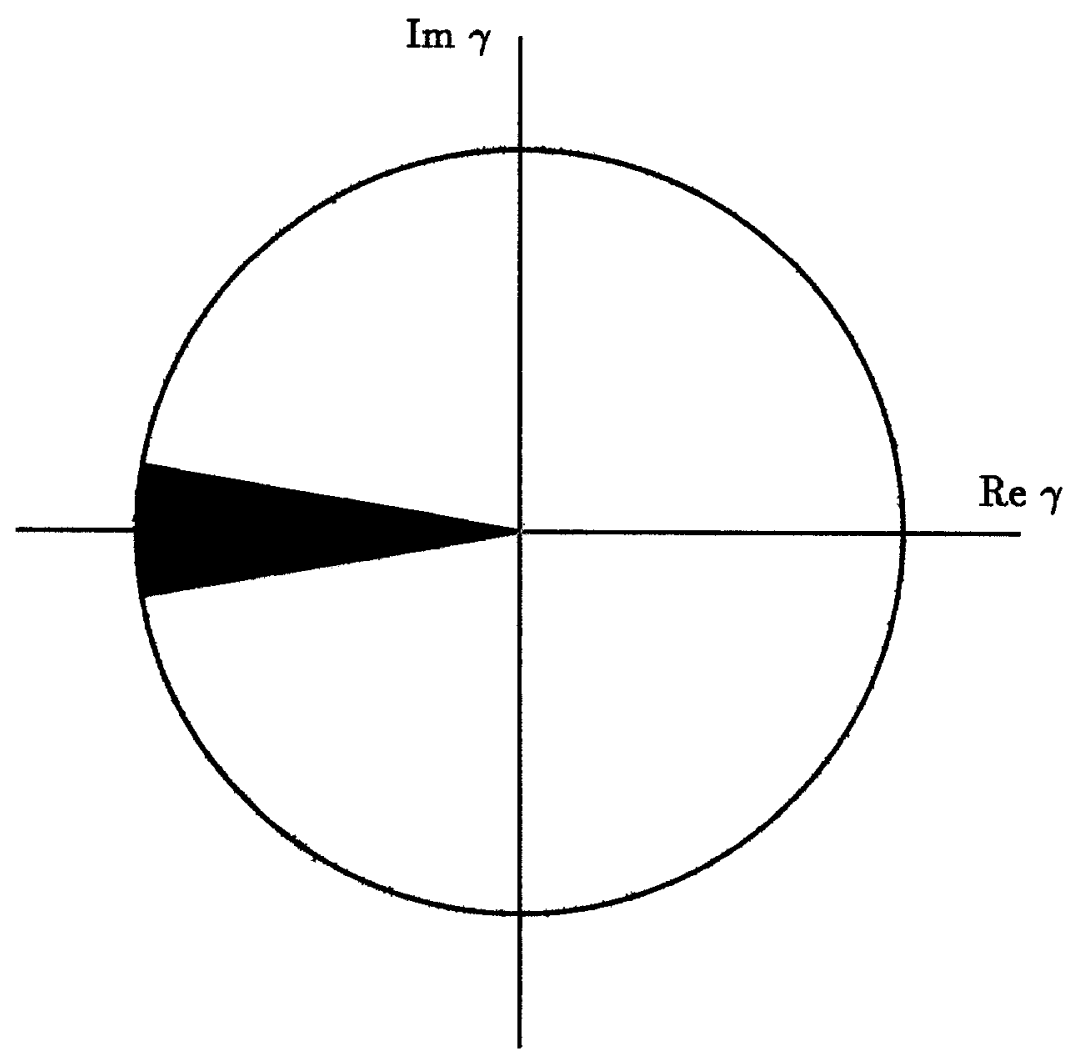




\section{TRL Algorithm FORTRAN Code}




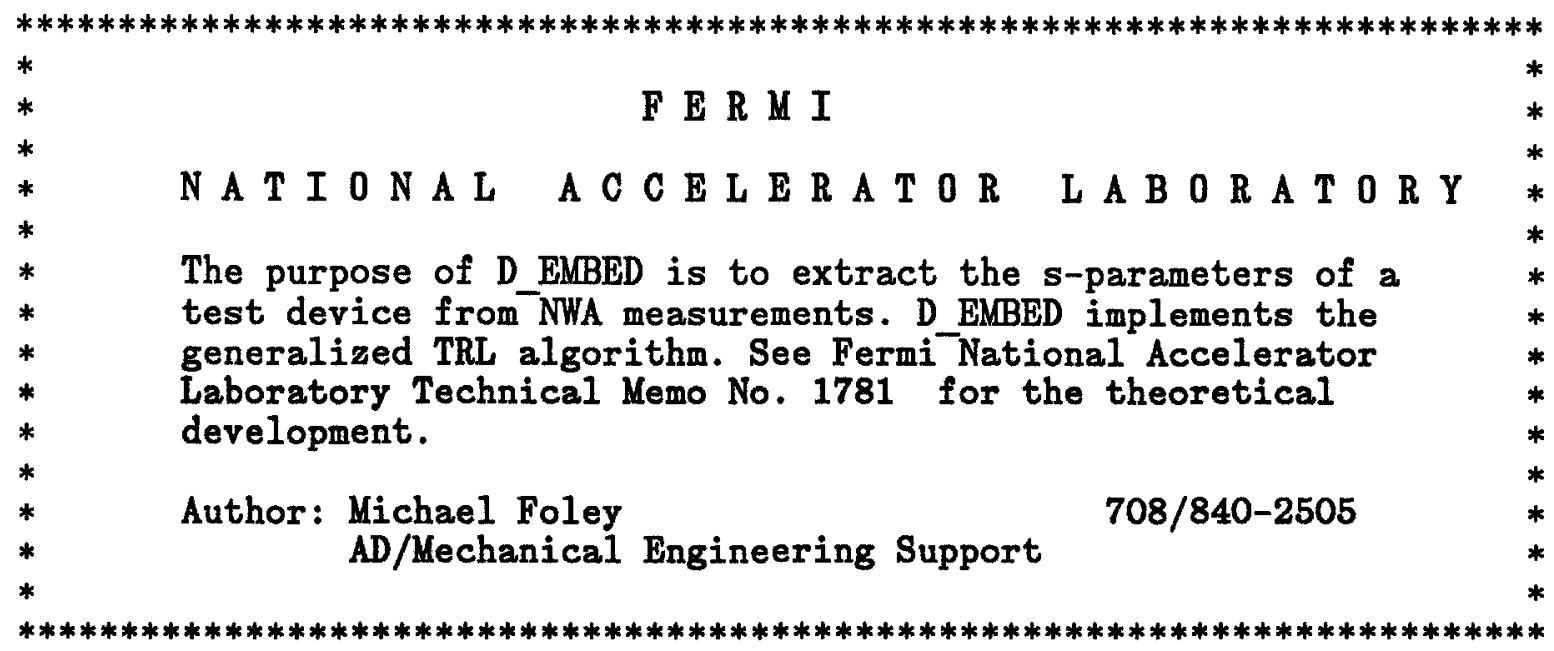

program d_embed

common/spard/sl1 $(2,2), \operatorname{sl2}(2,2), \operatorname{ss} 1(2,2), \operatorname{ss2}(2,2), \operatorname{sm}(2,2)$

common/sparc/s $(2,2)$

complex*16 s11, s12,ss1, ss2, sm, s

real $* 8$ freq, ratio

integer npoints, data_format

c Open the input data files and an output file

call file_open(ratio, npoints,data_format)

c De-embed the s-parameters of the test device

do $11 i=1$, npoints

call file read(freq, data format)

call s parameter (freq, ratio)

11

continue

write $(\overline{9} 6,100)$ freq, $s(1,1), s(2,1), s(1,2), s(2,2)$

close (unit $=91$ )

close (unit $=92$ )

close (unit=93)

close (unit $=94$ )

close (unit $=95$ )

close (unit=96)

100 format (1p9e12.4)

stop

end

subroutine file open(ratio, npoints, data format)

character*20 file line1, file line2,file short1,

$\mathbf{x}$

real*8 ratio

fille short2, file data, fille output

integer data format

character $* 10 \overline{0}$ title (22)

11 format(' ', 'Enter the name of the Line 1 calibration file (use single $x$ quotes to enclose the file name): ', \$)

accept*,file_line1 
12 format(' ', 'Enter the name of the Line 2 calibration file (use single $x$ quotes to enclose the file name):',\$)

accept*,file_line2

format(' ', 'Enter the name of the Short 1 calibration file (use singl xe quotes to enclose the file name):',\$)

accept*,file_short1

type 14

14 format(' ', 'Enter the name of the Short 2 calibration file (use singl xe quotes to enclose the file name): $\left.{ }^{\prime}, \$\right)$

accept*,file_short2

type 15

15 format(' ', 'Enter the name of the file containing the measured s-para' xmeters for the test device (use single quotes):',\$)

accept*,file_data

type 16

16 format(' ', 'Enter the name of the output file for the computed s-para xmeters for the test device (use single quotes):',\$)

accept*,file_output

17 format(' ', 'Enter the number of data points in the files:', \$) accept*, npoints

18 format(' ', 'Enter the ratio of the lengths of the two transmission $1 i$ xnes (L2/L1) - If Line1 is a direct connection enter $\left.0:^{\prime}, \$\right)$

accept*, ratio

type 18

format(' ', 'Is calibration file data in mag, arg format(1) or re,im xformat (2):',\$)

accept*, data_format

open (unit $=91$, file=file line1, status='old')

open (unit $=92$, file $=f i l e-1$ ine2, status='old')

open (unit $=93$, file $=f i l e-$ short 1 , status='old')

open (unit $=94$, file $=f i l e$ short2, status='old')

open (unit=95, file=file_data, status='old')

open (unit=96,file=file_output, status='new')

c Read data file headers

do $20 i=1,22$

$\operatorname{read}(91,100)$ title(i)

$\operatorname{read}(92,100)$ title $(i)$

$\operatorname{read}(93,100)$ title $(i)$

read $(94,100)$ title(i)

continue

$$
\operatorname{read}(95,100) \text { title }(i)
$$

100 format(a100)

return

end 
subroutine file read(freq, data format)

common/spard/s1 $(2,2), \operatorname{sl2}(2,2), \operatorname{ss} 1(2,2), \operatorname{ss} 2(2,2), \operatorname{sm}(2,2)$

complex*16 s11, s12,ss1, ss2, sm

real*8 freq

real*8 s11m, s11a, s21m, s21a, s12m, s12a, s22m, s22a

integer data_format

if (data_format.eq.1) then

Data in magnitude, argument format

$\operatorname{read}(91, *)$ freq, s11m,s11a,s21m,s21a, s12m,s12a,s22m,s22a $\operatorname{s11}(1,1)=\operatorname{dcmplx}(\mathbf{s} 11 \mathrm{~m} * \operatorname{dcosd}(\mathrm{s} 11 \mathrm{a}), \mathrm{s} 11 \mathrm{~m} * \mathrm{dsind}(\mathrm{s} 11 \mathrm{a}))$ s11 $(2,1)=\operatorname{dcmp} 1 \times(s 21 m * d \operatorname{cosd}(s 21 a)$, s21m*dsind (s21a) $\mathrm{s} 11(1,2)=$ dcmplx (s12m $*$ dcosd (s12a), s12m*dsind (s12a) $s 11(2,2)=d \operatorname{cmp} 1 x(s 22 m * d \cos d(s 22 a), s 22 m * d \operatorname{sind}(s 22 a))$

read (92,*) freq, s11m,s11a,s21m,s21a, s12m,s12a,s22m, s22a s12 $(1,1)=\operatorname{dcmp} 1 x(s 11 m * d \operatorname{cosd}(s 11 a), s 11 m * d s i n d(s 11 a))$ s12 (2,1)=dcmplx (s21m*dcosd (s21a), s21m*dsind (s21a)) $\operatorname{s12}(1,2)=d \operatorname{cmp} 1 x(\mathbf{s} 12 \mathrm{~m} * \operatorname{dcosd}(\mathbf{s} 12 \mathrm{a}), \mathbf{s 1 2 m} * \operatorname{dsind}(\mathbf{s} 12 \mathrm{a}))$ s12 $(2,2)=\operatorname{dcmplx}(\mathrm{s} 22 \mathrm{~m} * \operatorname{dcosd}(\mathrm{s} 22 \mathrm{a}), \mathrm{s} 22 \mathrm{~m} * \mathrm{dsind}(\mathrm{s} 22 \mathrm{a}))$

$\operatorname{read}(93, *)$ freq, s11m, s11a,s21m, s21a, s12m, s12a, s22m, s22a

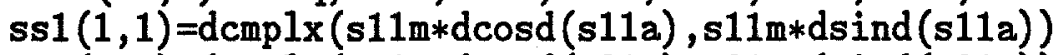
ss1 $(2,1)=\operatorname{dcmp} 1 x(s 21 m * d \operatorname{cosd}(s 21 a), s 21 m * d s i n d(s 21 a))$ ss $1(1,2)=\operatorname{dcmp} 1 x(s 12 m * d \operatorname{cosd}(s 12 a), s 12 m * d \operatorname{sind}(s 12 a))$ ss1 $(2,2)=\operatorname{dcmp} 1 x(s 22 m * \operatorname{dcosd}(s 22 a)$, s22m*dsind (s22a)

$\operatorname{read}(94, *)$ freq, s11m,s11a,s21m,s21a, s12m,s12a, s22m, s22a $\operatorname{ss} 2(1,1)=\operatorname{dcmplx}(\mathrm{s} 11 \mathrm{~m} * \operatorname{dcosd}(\mathrm{s} 11 \mathrm{a}), \mathrm{s} 11 \mathrm{~m} * \mathrm{dsind}(\mathrm{s} 11 \mathrm{a}))$ ss2 $(2,1)=\operatorname{dcmplx}(\operatorname{sim} * \operatorname{dcosd}(\mathrm{s} 21 \mathrm{a})$, s21m*dsind (s21a)) $\operatorname{ss} 2(1,2)=\operatorname{dcmp} 1 x(s 12 m * d \operatorname{cosd}(s 12 a)$, s12m*dsind $(s 12 a))$ ss $2(2,2)=\operatorname{dcmplx}(\mathrm{s} 22 \mathrm{~m} * \operatorname{dcosd}(\mathrm{s} 22 \mathrm{a}), \mathrm{s} 22 \mathrm{~m} * \mathrm{dsind}(\mathrm{s} 22 \mathrm{a}))$

$\operatorname{read}(95, *)$ freq, s11m,s11a,s21m,s21a, s12m, s12a, s22m, s22a $\operatorname{sm}(1,1)=\operatorname{dcmplx}(\operatorname{si1m} * \operatorname{dcosd}(\mathrm{s} 11 \mathrm{a}), \operatorname{s11} m * \operatorname{dsind}(\mathrm{s} 11 \mathrm{a}))$ $\operatorname{sm}(2,1)=\operatorname{dcmplx}(\operatorname{sim} m * \operatorname{dcosd}(s 21 a), s 21 m * d \operatorname{sind}(s 21 a))$ $\operatorname{sm}(1,2)=\operatorname{dcmplx}(\mathrm{s} 12 \mathrm{~m} * \mathrm{~d} \operatorname{cosd}(\mathrm{s} 12 \mathrm{a}), \mathrm{s} 12 \mathrm{~m} * \mathrm{dsind}(\mathrm{s} 12 \mathrm{a}))$ $\operatorname{sm}(2,2)=\operatorname{dcmp} 1 x(\sin m * \operatorname{dcosd}(\sin 2), \sin m * \operatorname{dsind}(s 22 a))$

else if (data_format.eq.2) then

Data in real, imaginary format

$\operatorname{read}(91,99)$ freq, $\operatorname{sl1}(1,1), \operatorname{sl1}(2,1), \operatorname{sl1}(1,2), \operatorname{sll}(2,2)$

$\operatorname{read}(92,99)$ freq, s12 $(1,1), \operatorname{si2}(2,1), \operatorname{si2}(1,2), s 12(2,2)$

read $(93,99)$ freq, ss1 $(1,1)$, ss1 $(2,1), \operatorname{ss} 1(1,2)$, ss1 $(2,2)$

read $(94,99)$ freq, ss2 $(1,1)$, ss2 $(2,1)$, ss2 $(1,2)$, ss2 $(2,2)$

$\operatorname{read}(95,99)$ freq, $\operatorname{sm}(1,1), \operatorname{sm}(2,1), \operatorname{sm}(1,2), \operatorname{sm}(2,2)$

endif

return

end

subroutine s_parameter (freq,ratio)

implicit complex $* 16 \quad(a-h, 0-z)$ 
common/spard/s11 $(2,2), \operatorname{sl2}(2,2), \operatorname{ss} 1(2,2), \operatorname{ss} 2(2,2), \operatorname{sm}(2,2)$

common/sparc/s $(2,2)$

dimension $p(2,2), q(2,2)$

complex*16 sl1, s12, ss 1 , ss 2 , sm

complex*16 p, q, s, lplus, lminus, gamma, z

real $* 8$ freq, ratio, power, garg

real*8 1pmag, lparg

Evaluate $P$ and $Q$ matrices

deltasl1=sl1 $(1,1) * s 11(2,2)-\operatorname{sl1}(2,1) * \operatorname{s} 11(1,2)$

deltas $12=s 12(1,1) * s 12(2,2)-s 12(2,1) * s 12(1,2)$

$p(1,1)=s 12(1,1) * s 11(2,2)-\operatorname{deltas} 12$

$\mathrm{p}(2,1)=\operatorname{sl1}(2,2)-\mathrm{s} 12(2,2)$

$p(1,2)=s 11(1,1) *$ deltasl2-s $12(1,1) *$ deltas 11

$\mathrm{p}(2,2)=\operatorname{sl1}(1,1) * \mathrm{~s} 12(2,2)-$ deltasl1

$q(1,1)=s 11(1,1) * s 12(2,2)-\operatorname{deltas} 12$

$q(2,1)=s 12(2,2) * \operatorname{deltas} 11-s 11(2,2) * \operatorname{deltas} 12$

$q(1,2)=s 12(1,1)-\operatorname{sll}(1,1)$

$q(2,2)=s 12(1,1) * s 11(2,2)-\operatorname{de} 1 \operatorname{tas} 11$

do $11 i=1,2$

do $11 j=1,2$

$$
\begin{aligned}
& p(i, j)=(1 . /(\operatorname{s11}(1,2) * \operatorname{sl2}(2,1))) * p(i, j) \\
& q(i, j)=(1 . /(\operatorname{sl1}(1,2) * s 12(2,1))) * q(i, j)
\end{aligned}
$$

continue

\section{continue}

c

Calculate the pair of roots (lplus and lminus) of the quadratic equation

$$
\mathrm{L} * * 2-\operatorname{Tr} \mathrm{P} * \mathrm{~L}+\mathrm{DELTAP}=0
$$

deltap $=p(1,1) * p(2,2)-p(2,1) * p(1,2)$

deltaq $=q(1,1) * q(2,2)-q(2,1) * q(1,2)$

lplus $=0.5 *((p(1,1)+p(2,2))+\operatorname{sqrt}((p(1,1)+p(2,2)) * * 2-4 . *$ deltap $))$

lminus $=0.5 *((\mathrm{p}(1,1)+\mathrm{p}(2,2))-\operatorname{sqrt}((\mathrm{p}(1,1)+\mathrm{p}(2,2)) * * 2-4 . * \operatorname{deltap}))$

1pmag=abs (1plus)

lparg $=57.2958 * \operatorname{atan} 2$ (dimag (1plus), dreal (1plus))

c Assign roots of quadratic equation to proper location in

c [L] matrix

if (1parg.gt.0.) then

$\mathrm{z}=1 \mathrm{plus}$

lplus=lminus

lminus $=\mathbf{z}$

endif 
ra11ra21 $=(1 \mathrm{plus}-\mathrm{p}(2,2)) / \mathrm{p}(2,1)$

ra12ra22 $=(1$ minus $-p(2,2)) / p(2,1)$

rb11rb12=(1plus-q(2,2) $) / q(1,2)$

rb21rb22=(1minus $-q(2,2)) / q(1,2)$

Evaluate reflections from Short 1 and Short 2 respectively

rho $a=s s 1(1,1)$

rho_b=ss2 $(2,2)$

alpha=(ra12ra22-rho a)/(rho a-ra11ra21)

beta $=($ rb21rb22+rho_b) $/($ rho_b+rb11rb12)

Calculate L1plus and L1minus

if (ratio.gt.1) then power=1./(ratio-1.)

1plus $=1$ plus $* *$ power

else

lminus $=1$ minus $* *$ power

endif

lplus $=1.0$

lminus $=1.0$

$\operatorname{ra22ra11=-sqrt}((\operatorname{ra11} \operatorname{ra} 21 *(1 . / \operatorname{si1}(1,1))-1) /$.

$((1 .-\operatorname{ra12ra} 22 *(1 . / \operatorname{sil}(1,1))) * \operatorname{ra11ra} 21 * * 2 *$

(alpha/beta)*(lminus/1plus)))

Select proper root by checking phase of reflect

gamma=ra22ra11*(rho a-ra12ra22)/(1.-rho_a*(1./ra11ra21))

garg=57.2958*atan2 (dimag (gamma), dreal (gäma))

if (abs (garg) . 1t .90.) then

endif ra22ra11=-ra22ra11

rb22rb11=(alpha/beta) $*$ ra11ra21*ra22ra11/rb11rb12

Evaluate appropriate s-parameters of $A$ and $B$ networks

sa11=ra12ra22

$\mathrm{sa22}=-1 . /($ ra11ra21 $*$ ra22ra11)

sa12_sa21 $=(1 . / \operatorname{ra22} \operatorname{ra11})-\operatorname{ra12ra22} /(\operatorname{ra22ra11} * \operatorname{ra11} \mathrm{ra21})$

sb11=1 . /(rb11rb12*rb22rb11)

sb22=-rb21rb22

sb21_sb12=(1./rb22rb11) -rb21rb22/(rb22rb11*rb11rb12)

sa21_sb21=-s11 $(2,1) *$ sa22*sb11*1plus*

$x-(1 .+($ alpha/beta $) *($ minus/1p1us $) *($ ra11ra21*ra22ra11)**2)

sa12_sb12=sa21_sb21*(s11 $(1,2) / \operatorname{si1}(2,1))$

Evaluate error terms for standard NWA error model

edf $=s a 11$

esf $=\mathbf{s a 2 2} * 1 \mathrm{plus}$

erf $=$ sa12_sa21*1plus 
elf $=$ sb11 $* 1$ plus

etf $=$ sa21_sb21*1plus

edr $=s b 22$

esr $=$ sb11 $*$ lplus

err $=$ sb21_sb12*1plus

elr=sa22*1plus

etr=sa12_sb12*lplus

c

Solve for de-embedded s-parameters of the test device

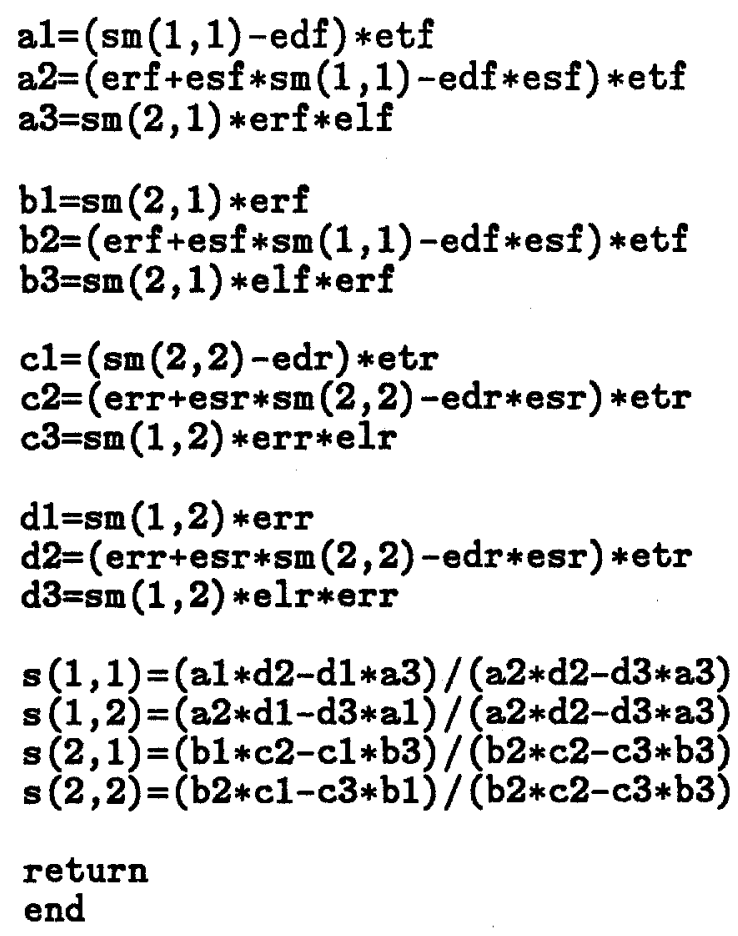




\section{E Impedance FORTRAN Code}


program z_calc

c Calculate magnitude and phase of impedance from de-imbedded s-parameters

complex*16 s11(1000), s21(1000), s12(1000), s22(1000), zz (1000)

complex $* 16$ deltas

real*8 freq (1000), zmag (1000), zphase (1000)

real*8 s11m,s11a,s21m,s21a, s12m, s12a,s22m, s22a

character $* 20$ data_file

type 8

8 format(', 'Enter name of s-parameter file (single quotes): '\$)

accept*, data_file

open (unit=91, file=data file, status='old')

open (unit=92, file='zmag. dat', status='new')

open (unit=93,file='zphase.dat', status='new')

type 11

11 format(' ', 'Enter number of data points:' ,\$)

accept*, npoints

do $12 i=1$, npoints

c

Data in real, imaginary format

$\operatorname{read}(91,100) \operatorname{freq}(i), s 11(i), s 21(i), s 12(i), s 22(i)$

Data in magnitude, phase(degrees) format

c

$\operatorname{read}(91, *)$ freq(i) , s11m,s11a,s21m, s21a,s12m, s12a,s22m, s22a

$s 11(i)=\operatorname{dcmp} 1 x(s 11 m * \operatorname{dcosd}(s 11 a)$, s11m*dsind (s11a))

s21 (i) =dcmplx (s21m*dcosd (s21a), s21m*dsind (s21a))

s12 (i) $=\operatorname{dcmp} 1 \times$ (s12m*dcosd (s12a), s12m*dsind (s12a))

$\mathbf{s} 22(i)=\operatorname{dcmp} 1 \times(s 22 m * \operatorname{dcosd}(s 22 a), \operatorname{s22m} * d \operatorname{sind}(s 22 a))$

c

12

$\operatorname{deltas}=s 11(i) * s 22(i)-s 21(i) * s 12(i)$

$\mathrm{zz}(i)=(1 .+\mathrm{s} 11(i)+\mathrm{s} 22(i)+\operatorname{de1tas}) /(2 . * \mathrm{~s} 21(i))$

$\mathrm{zz}(i)=266 . * \mathrm{zz}(i)$

continue

do 20 i=1, npoints

$\operatorname{zmag}(i)=\operatorname{abs}(\mathbf{z z}(i))$

20 continue $z$ phase $(i)=57.2958 * \operatorname{atan} 2(\operatorname{dimag}(z z(i)), \operatorname{dreal}(z z(i)))$

do $40 i=1$, npoints write $(92, *)$ freq(i), zmag (i)

write $(93, *)$ freq(i), zphase $(i)$

40

continue

100 format ( $9 \mathrm{e} 12.4)$

stop

end 